\title{
Combined Effects of an 3-Hydroxy-3-Methylglutaryl Coenzyme A Reductase Inhibitor and Angiotensin II Receptor Antagonist on Nitric Oxide Bioavailability and Atherosclerotic Change in Myocardial Infarction- Prone Watanabe Heritable Hyperlipidemic Rabbits
}

\author{
Toshio IMANISHI ${ }^{1)}$, Hideyuki IKEJIMA ${ }^{1)}$, Atsushi TSUJIOKA ${ }^{1)}$, Akio KUROI ${ }^{1)}$, \\ Katsunobu KOBAYASHI ${ }^{1)}$, Masashi SHIOMI ${ }^{2)}$, Yasuteru MURAGAKI ${ }^{3)}$, \\ Seiichi MOCHIZUKI ${ }^{4)}$, Masami GOTO ${ }^{4}$, Kiyoshi YOSHIDA ${ }^{5)}$, and Takashi AKASAKA ${ }^{1)}$
}

\begin{abstract}
We investigated the effects of co-administration of 3-hydroxy-3-methylglutaryl coenzyme A (HMG-CoA) reductase inhibitor and angiotensin II type 1 receptor blocker (ARB) on nitric oxide (NO) bioavailability in genetically hyperlipidemic rabbits with our newly developed NO sensor. A total of 36 myocardial infarctionprone Watanabe heritable hyperlipidemic (WHHLMI) rabbits equally derived ( $n=6$ per group) were treated with 1) vehicle (control), 2) hydralazine (15 mg/kg/d), 3) the HMG-CoA reductase inhibitor pitavastatin (P: 0.5 $\mathrm{mg} / \mathrm{kg} / \mathrm{d})$, 4) the ARB valsartan ( $\mathrm{V}: 5 \mathrm{mg} / \mathrm{kg} / \mathrm{d})$, and 5) pitavastatin + valsartan $(P+V)$ together without or 6) with $N^{G}$-nitro-L-arginine methyl ester (L-NAME) for 8 weeks. After treatment, acetylcholine (ACh)-induced NO production was measured as a surrogate for endothelium protective function, and vascular peroxynitrite (a product of superoxide and NO) was measured for assessing dysfunctional endothelial NO synthase activity. Plaque area was quantified by histology as well as optical coherence tomography (OCT). Intra-aortic infusion of ACh produced an increase in plasma NO concentration, which was significantly greater with all drug treatments than with the control. $\mathrm{P}+\mathrm{V}$ increased $\mathrm{ACh}$-induced $\mathrm{NO}$ by $4.1 \mathrm{nmol} / \mathrm{L}$ significantly more than either $P$ or $V$ singly. The vascular peroxynitrite concentration was $1.6 \mathrm{pmol} / \mathrm{mg}$ protein in the control group and significantly less than those in the P- and V-monotherapy-groups. The lowest peroxynitrite concentration was observed in the $\mathrm{P}+\mathrm{V}$ group $(0.4 \mathrm{pmol} / \mathrm{mg}$ protein), which was significantly lower than those in the $\mathrm{P}$ - and the V-monotherapy-groups. OCT and histology of the thoracic aorta revealed that the plaque area decreased significantly more with the combination than with the monotherapy. In conclusion, the combined treatment with an HMG-CoA reductase inhibitor and an ARB may have additive protective effects on endothelial function as well as atherosclerotic change. (Hypertens Res 2008; 31: 1199-1208)
\end{abstract}

Key Words: nitric oxide, endothelium, statin, angiotensin II type 1 receptor blocker, nitrative stress

From the ${ }^{1)}$ Department of Cardiovascular Medicine and ${ }^{3)}$ Department of Pathology, Wakayama Medical University, Wakayama, Japan; ${ }^{2}$ Institute for Experimental Animals, Kobe University School of Medicine, Kobe, Japan; and ${ }^{4}$ Department of Medical Engineering and ${ }^{5}$ Division of Cardiology, Kawasaki Medical School, Kurashiki, Japan.

Address for Reprints: Toshio Imanishi, M.D., Ph.D., Department of Cardiovascular Medicine, Wakayama Medical University, 811-1, Kimiidera, Wakayama 641-8510, Japan. E-mail: t-imani@wakayama-med.ac.jp

Received October 30, 2007; Accepted in revised form December 28, 2007. 


\section{Introduction}

An activated renin-angiotensin system and hypercholesterolemia are well-established risk factors for coronary artery disease (1). Approximately $40 \%$ of patients with hypertension have hypercholesterolemia (2). However, hypertension is an important risk factor in patients with increased cholesterol (3). Pharmacological treatment of both risk factors by 3hydroxy-3-methylglutaryl coenzyme A (HMG-CoA) reductase inhibitors (statins) and angiotensin II type 1 (AT1) receptor blockers (ARBs) reduce end-organ damage and cardiovascular mortality and morbidity (4, 5). However, beyond the cholesterol- and blood pressure-lowering effects, there may be additional mechanisms underlying the protective effects of both therapies, including nitric oxide (NO) availability, and reduced oxidative stress.

Reliable evaluation of NO production would be particularly important, for instance, for early vascular abnormalities. Recently, this group has developed a catheter-type NO sensor, which can directly measure intra-arterial NO concentration in vivo and evaluate detailed in vivo endothelial function $(6,7)$. The catheter-type NO sensor has potential use in the study of the pharmacological modulation of NO, enabling the measurement of the basal and acethylcholine (ACh)-induced NO production in the aorta of experimental animal models. With the sensor, we have shown for the first time that chronic treatment with angiotensin II (Ang II) impairs endothelial function by reducing basal and ACh-induced plasma NO concentration in anesthetized New Zealand White rabbits (8).

Statins and ARBs are well-established therapeutic agents in the treatment of hypertension, hypercholesterolemia, and coronary artery disease (9). Growing evidence supports the idea that the two medications have synergistic beneficial effects. However, the effects of these agents on the bioavailability of NO and vascular remodeling have not been investigated in detail. In the present study, the effects of a statin, pitavastatin, an ARB, valsartan, and their combination on NO bioavailability as measured with our sensor, on atherosclerotic change, and on the vascular peroxynitrite level were investigated in myocardial infarction-prone Watanabe heritable hyperlipidemic (WHHLMI) rabbits.

\section{Methods}

\section{Catheter-Type NO Sensor}

The integrated architecture and performance of the cathetertype NO sensor have been described previously (6-8). In brief, an NO sensor (amino-700 XL; $700 \mu \mathrm{m}$ in diameter at the detection tip; Innovative Instruments), was used to measure the oxidative current of NO with an NO monitor (model inNO-T; Innovative Instruments). For each sensor, the baseline (zero level) was set arbitrarily in the amperometric method and calibrated with NO-saturated pure water. The change in the current from the baseline was expressed as the change in NO concentration ( $\mathrm{nmol} / \mathrm{L})$.

\section{Animal Preparation}

This group complied with the Guide for the Care and Use of Laboratory Animals published by the US National Institutes of Health. In addition, the study protocol was approved by the Institutional Animal Care and Use Committee of Wakayama Medical University. Thirty-six WHHLMI rabbits (3 months old) were randomized into one of six groups. Each group received oral administration of vehicle, $0.5 \%$ carboxymethylcellulose sodium (control group), $10 \mathrm{mg} / \mathrm{kg} / \mathrm{d}$ of hydralazine (hydralazine group), $0.5 \mathrm{mg} / \mathrm{kg} / \mathrm{d}$ of pitavastatin (pitavastatin group), $5 \mathrm{mg} / \mathrm{kg} / \mathrm{d}$ of valsartan (valsartan group), and $0.5 \mathrm{mg} /$ $\mathrm{kg} / \mathrm{d}$ of pitavastatin plus $5 \mathrm{mg} / \mathrm{kg} / \mathrm{d}$ of valsartan (pitavastatinvalsartan group) together with or without $15 \mathrm{mg} / \mathrm{kg} / \mathrm{d}$ of $N^{\mathrm{G}}$ nitro-L-arginine methyl ester (L-NAME) daily by gavage for 8 weeks. In our previous studies, $5 \mathrm{mg} / \mathrm{kg} / \mathrm{d}$ of valsartan significantly reversed the Ang II-induced increase in mean arterial pressure almost to the control level in an Ang II-infusion rabbit model (8). Therefore, we used this dose in the present study. On the other hand, pitavastatin was administered at a dose of $0.5 \mu \mathrm{g} / \mathrm{kg} / \mathrm{d}$ in the present study. In previous experiments where the same dose was administered in drinking water, a plasma drug concentration of approximately $40 \mathrm{ng} /$ $\mathrm{mL}$ was maintained (10). The area under the concentration curve (AUC) from $0-24 \mathrm{~h}$ was $1 \mu \mathrm{g} / \mathrm{h} / \mathrm{mL}$, which was close to the $C_{\max }$ attained after the administration of $2 \mathrm{mg}$ to humans, and thus we inferred that the drug concentration in this experiment was equivalent to the attained in humans. Rabbits were anesthetized with xylazine (10 mg/kg intramuscularly), ketamine (50 mg/kg intramuscularly), and pentobarbital sodium (10 mg/kg intravenous), followed by administration of heparin (1,000 units intravenous) as an anticoagulant. A catheter for ACh and $N^{\mathrm{G}}$-methyl-L-arginine (L-NMMA) infusion was placed in the aortic arch from the external carotid artery. The NO sensor was inserted through the left femoral artery and placed in the abdominal aorta. Aortic blood pressure was simultaneously monitored through a stiff cannula with a strain gauge pressure transducer (Nihon Kohden, Tokyo, Japan). Blood samples were collected in EDTA $(1 \mathrm{mg} / \mathrm{mL})$ from the ear artery. Plasma was separated and analyzed for total cholesterol (TC) and triglyceride (TG) concentrations using a commercially available kit (Wako Pure Chemical Industries, Osaka, Japan).

\section{Measurement of NO Production}

$\mathrm{ACh}(4 \mu \mathrm{g} / \mathrm{kg} / \mathrm{min})$ was administered for $5 \mathrm{~min}$ to measure endothelium-dependent NO production. Basal NO production was measured after infusion of an NO synthase (NOS) inhibitor, L-NMMA $(5 \mathrm{mg} / \mathrm{kg})$ at $1 \mathrm{~mL} / \mathrm{min}$ for $10 \mathrm{~min}$. The plasma NO concentration in the abdominal aorta was monitored over the entire time course. We measured ACh-induced 
as well as L-NMMA-induced plasma NO production as the peak change in the current from the baseline, and this value taken as the change in $\mathrm{NO}$ concentration $(\mathrm{nmol} / \mathrm{L})$.

\section{Vessel Preparation}

The aorta was placed in chilled, modified Krebs/HEPES buffer (buffer A composition [mmol/L]: $\mathrm{NaCl} 99.01 ; \mathrm{KCl}$, 4.69; $\mathrm{CaCl}_{2}, 1.87 ; \mathrm{MgSO}_{4}, 1.20 ; \mathrm{K}_{2} \mathrm{HPO}_{4}, 1.03 ; \mathrm{NaHCO}_{3}$, 25.0; Na-HEPES, 20.0; and glucose, 11.1; pH 7.4), cleaned of excessive adventitial tissue, and cut into 5-mm ring segments.

\section{Estimation of NADH/NADPH Oxidase Activity in Vessel Homogenates}

Aortic segments were placed in chilled buffer A as described above. A $10 \%$ vessel homogenate was prepared in $50 \mathrm{nmol} / \mathrm{L}$ phosphate buffer by homogenizing aortic segments in a glassto-glass motorized homogenizer. The homogenizing buffer (buffer B) was a 50-mmol/L phosphate buffer containing 0.01 $\mathrm{mmol} / \mathrm{L}$ EDTA. The homogenate was subjected to low speed centrifugation $(1,000 \times g)$ for $10 \mathrm{~min}$ to remove unbroken cells and debris. Aliquots of the supernatant were then added to scintillation vials containing lucigenin $(5 \mu \mathrm{mol} / \mathrm{L})$ in $2 \mathrm{~mL}$ of buffer B. Chemiluminescence over the ensuing $5 \mathrm{~min}$ in response to addition of either NADH or NADPH (both 100 $\mu \mathrm{mol} / \mathrm{L}$ ) was recorded by a luminometer (LB9505; Berthold Technologies, Germany). Values were standardized to the amount of protein present, which was measured using a commercially available kit (DC Protein Assay; BioRad Laboratories, Hercules, USA).

\section{Measurement of Vascular Nitrotyrosine}

Peroxynitrite is a strong oxidant formed in the reaction between NO and superoxide under atherosclerotic stimuli. The peroxynitrite subsequently reacts with proteins resulting in nitrotyrosine. As a stable end product of peroxynitritemediated oxidation/nitration, nitrotyrosine can be used as a surrogate index of in vivo uncoupled NOS-dependent damage. Binding of vascular nitrotyrosine to proteins was measured using an NWLSS ${ }^{\mathrm{TM}}$ nitrotyrosine enzyme-linked immunoassay (ELISA) kit (Northwest Life Science Specialties, LLC, Vancouver, Canada) according to the manufacturer's protocol (11).

\section{Western Blotting}

Aorta samples were homogenized in ice-cold RIPA buffer ( $150 \mathrm{mmol} / \mathrm{L} \mathrm{NaCl}, 50 \mathrm{mmol} / \mathrm{L}$ Tris-HCl, $5 \mathrm{mmol} / \mathrm{L}$ EDTA, $1 \% \mathrm{v} / \mathrm{v}$ Nonidet P-40, 0.5\% w/v deoxycholate, $10 \mathrm{mmol} / \mathrm{L}$ phenylmethylsulfonyl fluoride, $2 \mu \mathrm{g} / \mathrm{mL}$ aprotinin, and $2 \mu \mathrm{g} /$ $\mathrm{mL}$ leupeptin). Aortic extracts (30 $\mu \mathrm{g}$ protein per lane) were mixed with sample loading buffer and separated on a $12 \%$ SDS-polyacrylamide gel. Proteins were electro-transferred to
Table 1. Time Course of Mean Atrial Pressure

\begin{tabular}{lccc}
\hline \multirow{2}{*}{ Groups } & \multicolumn{3}{c}{ Mean atrial pressure } \\
\cline { 2 - 4 } & Basal & 4 week & 8 week \\
\hline Control & $72.3 \pm 1.4$ & $74.9 \pm 2.2$ & $76.6 \pm 1.5$ \\
Hydralazine & $73.1 \pm 1.6$ & $71.1 \pm 1.8$ & $70.8 \pm 1.9$ \\
Pitavastatin & $74.4 \pm 2.0$ & $73.5 \pm 2.4$ & $73.2 \pm 2.9$ \\
Valsartan & $72.5 \pm 1.7$ & $70.9 \pm 1.9$ & $70.5 \pm 1.7$ \\
Pitavastatin+valsartan & $73.0 \pm 1.6$ & $70.2 \pm 1.8$ & $69.4 \pm 2.2$ \\
\hline
\end{tabular}

Data are the mean \pm SEM.

polyvinylidene fluoride (PVDF) membranes (Immun-Blot ${ }^{\circledR}$ $0.2 \mu \mathrm{m}$; Bio-Rad Laboratories) and bands were detected with a chemiluminescence assay (ECL Plus Amersham, Buckinghamshire, UK), using primary antibodies for endothelial NOS (eNOS; Signal Transduction Laboratories, Cell Signaling Technology, Beverly, USA). The profile of each band was plotted using images published by the National Institutes of Health (NIH) and the densitometric band intensity was determined.

\section{Echocardiography}

Cardiac function was measured by echocardiography (model Prosound 5500; ALOKA Inc., Tokyo, Japan) with the rabbits hand-held in the conscious state. A two-dimensional, shortaxis view of the left ventricle was obtained at the level of the papillary muscles. M-mode tracings were recorded through the anterior and posterior left ventricular (LV) walls. LV enddiastolic diameter (LVEDD) and LV ejection fraction $(\% \mathrm{EF})$ were measured from three consecutive cardiac cycles.

\section{Optical Coherence Tomography Imaging}

The plaque area of the thoracic aorta was evaluated with optical coherence tomography (OCT) imaging. After the animals were sacrificed, the aorta from the aortic arch to the lower thoracic region was removed. The surrounding soft tissues were dissected from each specimen. The branches were tied off with sutures, and the distal end of the artery was plugged with a large cork. A 7F sheath was sewn into the proximal end of the artery to complete the closed system. Saline $(0.9 \%)$ kept at a temperature of $37^{\circ} \mathrm{C}$ was infused through the side arm of the sheath. The pressure inside the artery was maintained at a physiologic level $(60-80 \mathrm{mmHg})$ with a syringemanometer connected to the infusion pump. An intravascular OCT catheter (ImageWire; LightLab Imaging, Westford, USA) was inserted through the sheath. Serial images of the OCT were obtained using an automatic pullback device at a rate of $0.5 \mathrm{~mm} / \mathrm{s}$. The obtained OCT images were processed and analyzed using appropriate software from LightLab Imaging Inc. for off-line analysis. According to the previous reports (12), lipid was semiquantified as the number of involved quadrants on the cross-sectional OCT image. The 
Table 2. Lipidemic Effects of Treatment in WHHLMI Rabbit

\begin{tabular}{|c|c|c|c|c|}
\hline \multirow{2}{*}{ Groups } & \multicolumn{2}{|c|}{$\mathrm{TC}(\mathrm{mg} / \mathrm{dL})$} & \multicolumn{2}{|c|}{ TG (mg/dL) } \\
\hline & Basal & 8 week & Basal & 8 week \\
\hline Control & $886 \pm 46$ & $844 \pm 39$ & $288 \pm 16$ & $281 \pm 15$ \\
\hline Hydralazine & $891 \pm 42$ & $855 \pm 40$ & $292 \pm 17$ & $288 \pm 13$ \\
\hline Pitavastatin & $876 \pm 42$ & $563 \pm 21 *$ & $282 \pm 14$ & $264 \pm 11$ \\
\hline Valsartan & $851 \pm 43$ & $832 \pm 33$ & $276 \pm 15$ & $269 \pm 14$ \\
\hline Pitavastatin + valsartan & $878 \pm 38$ & $544 \pm 19 *$ & $283 \pm 15$ & $257 \pm 13$ \\
\hline
\end{tabular}

Data are the mean \pm SEM. WHHLMI, myocardial infarction-prone Watanabe heritable hyperlipidemic; TC, total cholesterol; TG, triglyceride. ${ }^{*} p<0.01 v s$. Control.

Table 3. Cardiac Function in WHHLMI Rabbit

\begin{tabular}{lccccc}
\hline \multirow{2}{*}{ Groups } & \multicolumn{2}{c}{ LVEDD $(\mathrm{mm})$} & & \multicolumn{2}{c}{ LVEF $(\%)$} \\
\cline { 2 - 5 } \cline { 5 - 6 } Control & Basal & 8 week & $11.1 \pm 0.9$ & Basal & $70.9 \pm 3.8$ \\
Hydralazine & $10.9 \pm 0.8$ & $10.8 \pm 1.2$ & & $73.6 \pm 4.5$ & $68.1 \pm 4.4$ \\
Pitavastatin & $10.6 \pm 1.1$ & $10.9 \pm 1.3$ & $69.2 \pm 4.8$ & $72.6 \pm 4.6$ \\
Valsartan & $10.8 \pm 0.8$ & $11.2 \pm 1.2$ & & $71.3 \pm 4.1$ & $70.1 \pm 4.2$ \\
Pitavastatin+valsartan & $10.5 \pm 0.9$ & $11.3 \pm 1.1$ & & $72.7 \pm 4.4$ & $74.0 \pm 3.6$ \\
\hline
\end{tabular}

Data are mean \pm SEM. WHHLMI, myocardial infarction-prone Watanabe heritable hyperlipidemic; LVEDD, left ventricular end-diastolic diameter; LVEF, left ventricular ejection fraction.

lipid-rich plaque was defined as lipid content in more than 2 quadrants in any of the images. OCT-identified plaque lesions were also quantified as the degree of arc in the involved segments in the cross-sectional OCT image along the thoracic aorta (12).

\section{Histological Analysis}

Each artery was pressure-fixed in $10 \%$ neutral buffered formalin for $48 \mathrm{~h}$. After the fixation and standard paraffin embedding, serial cross sections were processed for general histological staining with hematoxylin-eosin. The method for determining the intimal area/medial area ratio of the thoracic aorta, which is used as a measure of atherosclerotic burden, has been previously described (13). Six sections from each rabbit aorta were used to determine the intimal area/medial area ratio.

\section{Statistical Analysis}

All data were expressed as the mean \pm SEM. Differences between groups were analyzed by ANOVA followed by Scheffe's test and were considered to be significant when the $p$ value was less than 0.05 .

\section{Results}

\section{Calibration of Sensors}

The NO sensors were shown to be stable and to have high specificity to NO without responding to any change in oxygen concentrations, infusion of ACh, L-NMMA, or mixing solution (data not shown). The mean peak response with NO concentration was $365 \pm 11 \mathrm{pA} / \mathrm{nmol} / \mathrm{L}$ among the seven sensors in the present study. The value was comparable to the values obtained with the original sensor ( 6 ).

\section{Hemodynamics, Lipid Profiles, and Echocardio- graphic Data after 8-Week Treatment}

The mean atrial pressure (MAP), heart rate, and body mass were compared among experimental groups as shown in Table 1. MAP was slightly but not significantly lower after both valsartan and the pitavastatin-valsartan combination than that of the control group during 4- and 8-week treatment. Neither heart rate nor body weight differed between any drug treatment and the control. The TC and TG concentrations of both groups were similar at the beginning of the experiment. At 8 weeks, we observed significant differences among groups in TC concentration (Table 2). That is, the TC concentration was significantly lower in the pitavastatin group and pitavastatin-valsartan combination group than in the control or hydralazine group. On the other hand, neither LVEDD nor 
$\mathbf{A}$
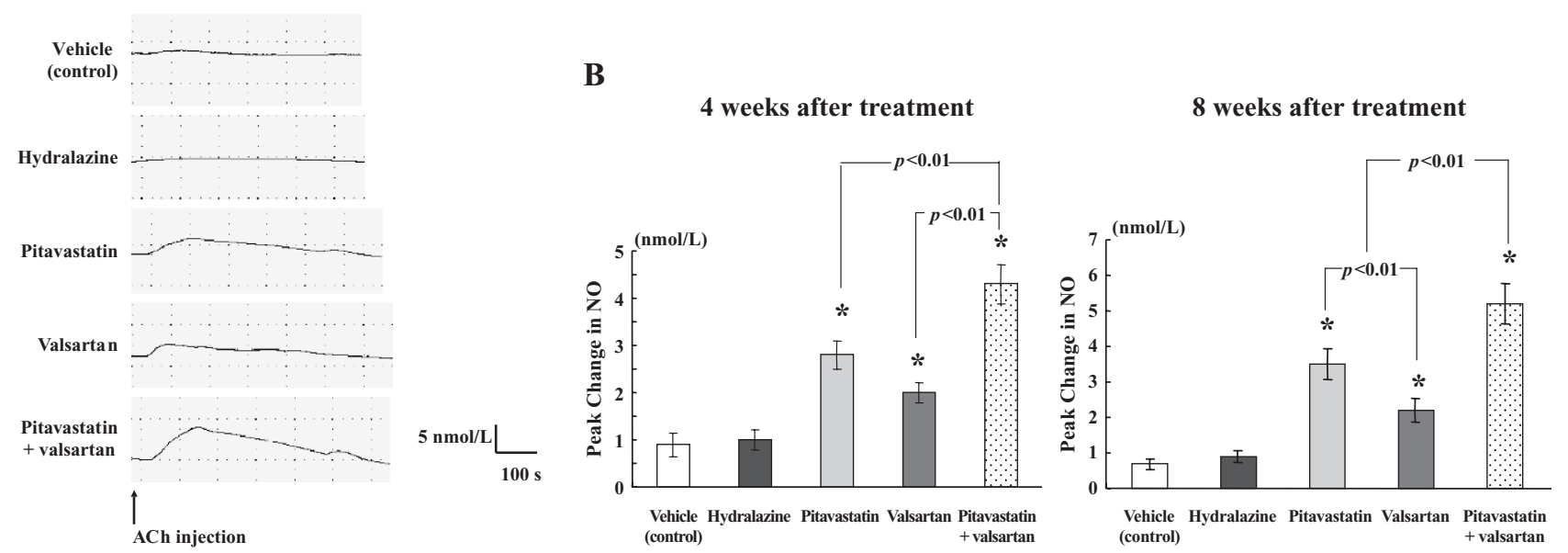

Fig. 1. Acetylcholine (ACh)-induced plasma NO production in the aorta of WHHLMI rabbits. A: Typical change in plasma NO concentration induced by $4 \mu \mathrm{g} / \mathrm{kg} / \mathrm{min}$ of ACh infusion (5 min) into the thoracic aorta among groups of WHHLMI rabbits $(n=6$ each) treated with vehicle (control), hydralazine $(10 \mathrm{mg} / \mathrm{kg} / \mathrm{d})$, pitavastatin $(0.5 \mathrm{mg} / \mathrm{kg} / \mathrm{d})$, valsartan $(5 \mathrm{mg} / \mathrm{kg} / \mathrm{d})$, and a combination of pitavastatin and valsartan for 8 weeks. B: Mean NO concentration change (nmol/L) at the peak response to ACh in five groups of rabbits treated with the above drugs for 4 (left) and 8 weeks (right) ( $n=6$ each). Data are shown as the mean $\pm S E M$. ${ }^{*} p<0.01$ vs. control.
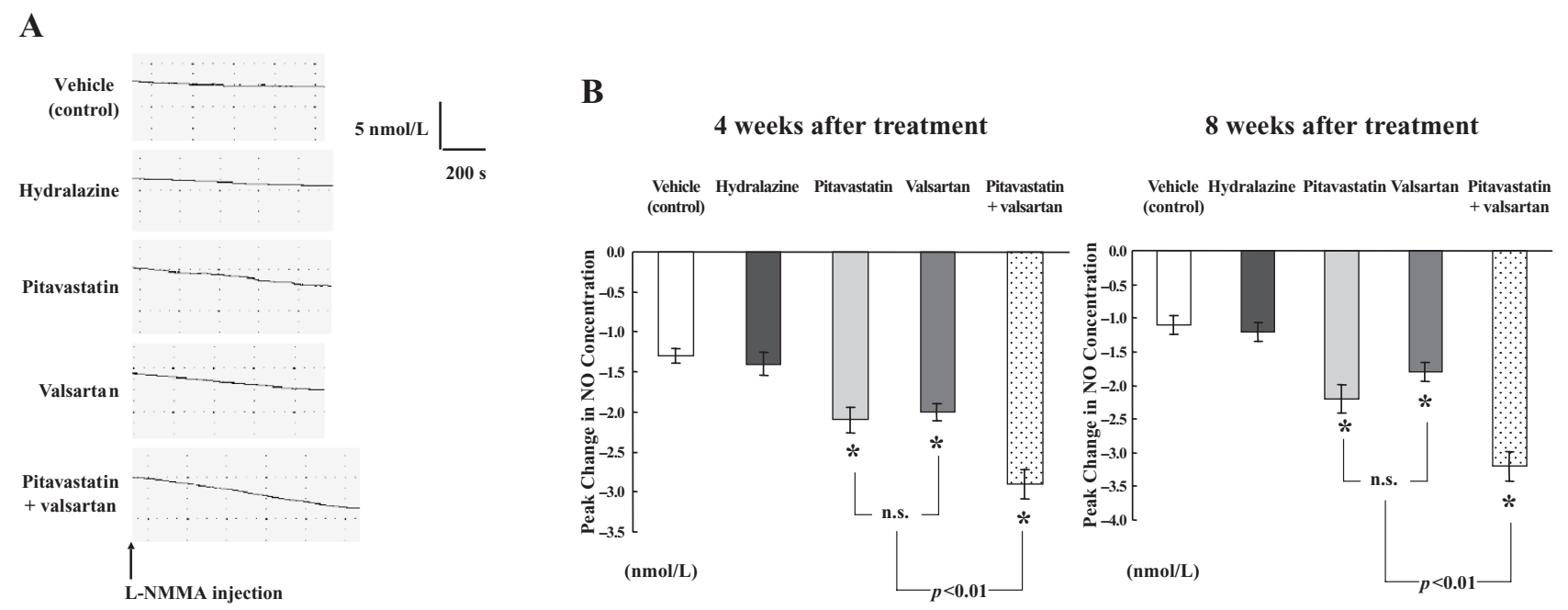

Fig. 2. Basal plasma NO concentration. A: Typical changes in plasma NO concentration of the thoracic aorta under L-NMMA infusion (10 min) among groups of WHHLMI rabbits treated with vehicle (control), hydralazine (10 $\mathrm{mg} / \mathrm{kg} / \mathrm{d})$, pitavastatin $(0.5$ $\mathrm{mg} / \mathrm{kg} / \mathrm{d})$, valsartan $(5 \mathrm{mg} / \mathrm{kg} / \mathrm{d})$, and the combination of pitavastatin and valsartan for 8 weeks. B: Mean basal plasma NO concentration of the thoracic aorta at the peak response to $5 \mathrm{mg} / \mathrm{kg}$ of L-NMMA infusion (10 $\mathrm{min}$ ) in five groups of rabbits treated with the above drugs for 4 (left) and 8 weeks (right) ( $n=6$ each). Data are shown as the mean $\pm S E M(n=6) .{ }^{*} p<0.01$ vs. control.

LVEF differed significantly between any drug treatment and the control (Table 3).

\section{ACh-Induced Increase in NO Synthesis after the 8-Week Treatments}

Endothelial function was monitored with ACh-induced NO synthesis (Fig. 1A). Intra-aortic infusion of ACh $(4 \mu \mathrm{g} / \mathrm{kg} / \mathrm{min}$ for $5 \mathrm{~min}$ ) produced an increase in plasma NO concentration, which was significantly greater with all the drug treatments, except for hydralazine, compared with the control $(p<0.01)$. The ACh-induced plasma NO concentration in the pitavastatin-treatment group was significantly higher than that in the valsartan-treatment group. The combination of pitavastatin and valsartan increased ACh-induced NO by about 4.1 $\mathrm{nmol} / \mathrm{L}$, which was significantly greater than the level in the 

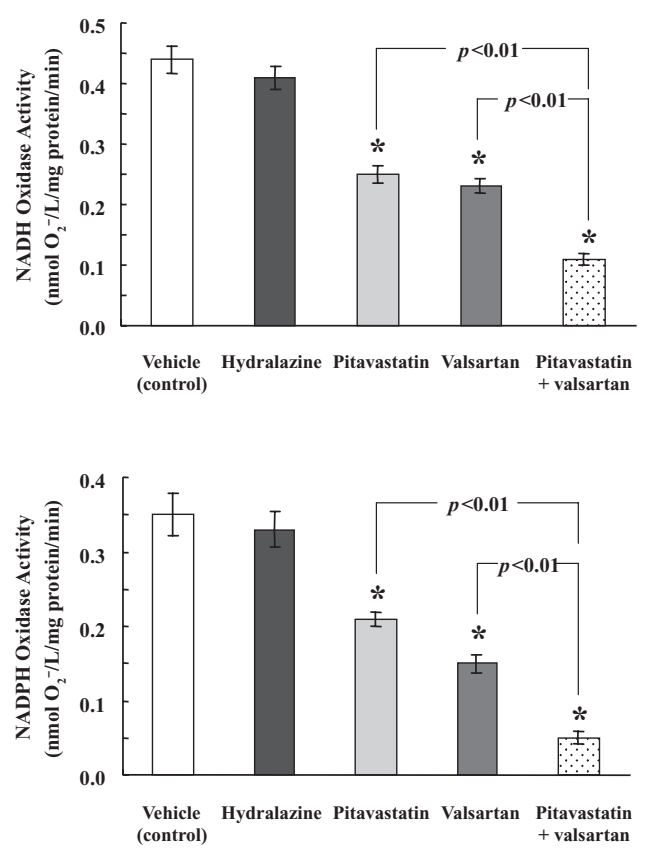

Fig. 3. NADH and NADPH oxidase activity in aortic homogenates after 8-week treatment with vehicle (control), hydralazine $(10 \mathrm{mg} / \mathrm{kg} / \mathrm{d})$, pitavastatin $(0.5 \mathrm{mg} / \mathrm{kg} / \mathrm{d})$, valsartan $(5 \mathrm{mg} / \mathrm{kg} / \mathrm{d})$, and pitavastatin and valsartan in combination. Data are expressed as the mean $\pm \operatorname{SEM}(n=6) .{ }^{*} p<0.01$ vs. control.

pitavastatin- or valsartan-monotherapy-group (Fig. 1).

\section{Basal NO Synthesis after 8-Week Treatments}

The effect of 8 weeks of treatment on the local basal NO concentration was evaluated by decreased $\mathrm{NO}$ concentration in the presence of $5 \mathrm{mg} / \mathrm{kg}$ of L-NMMA (Fig. 2). All the drug treatments, except for hydralazine, significantly increased the basal NO concentration more than that of the control group $(p<0.01)$. That is, the decrease in the basal plasma NO concentration by L-NMMA infusion was significantly higher with the pitavastatin-valsartan combination $(-3.4 \pm 0.2$ $\mathrm{nmol} / \mathrm{L})$ than with either pitavastatin $(-2.2 \pm 0.2 \mathrm{nmol} / \mathrm{L})$ or valsartan $(-1.8 \pm 0.1 \mathrm{nmol} / \mathrm{L})$ alone ( $p<0.01$ for both).

\section{Vascular Reactive Oxygen Species}

The activities of the NADH- and NADPH-dependent oxidases were both significantly lower in the drug-treated groups, except for the hydralazine group, than in the control group, and the combination treatment with pitavastatin and valsartan produced a significantly lower level of oxidase activity than either pitavastatin or valsartan alone (Fig. 3).

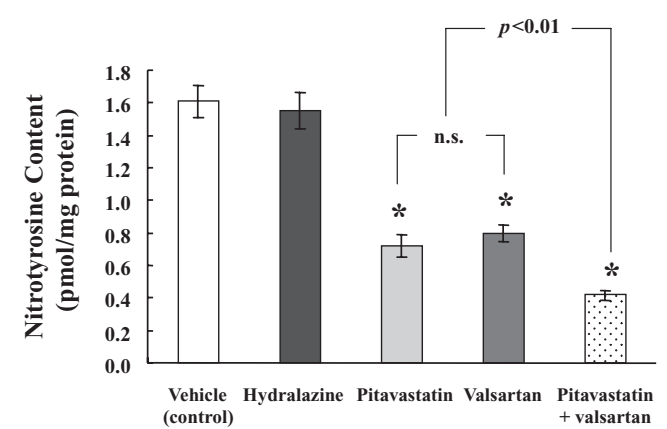

Fig. 4. Vascular nitrotyrosine content after 8-week treatment with vehicle (control), hydralazine (10 $\mathrm{mg} / \mathrm{kg} / \mathrm{d})$, pitavastatin $(0.5 \mathrm{mg} / \mathrm{kg} / \mathrm{d})$, valsartan $(5 \mathrm{mg} / \mathrm{kg} / \mathrm{d})$, and pitavastatin and valsartan in combination. Data are expressed as the mean $\pm \operatorname{SEM}(n=6)$. ${ }^{*} p<0.01$ vs. control.
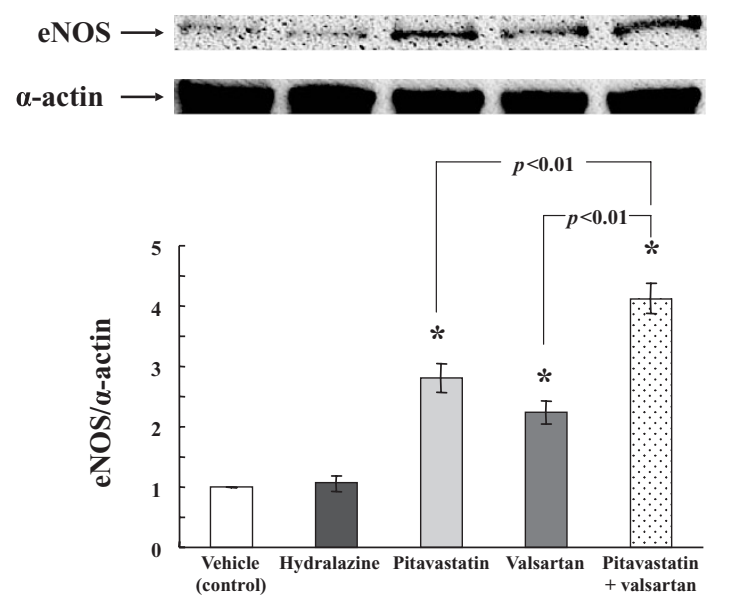

Fig. 5. Representative endothelial nitric oxide synthase (eNOS) protein expression in the thoracic aorta after 8-week treatment with vehicle (control), hydralazine $(10 \mathrm{mg} / \mathrm{kg} / \mathrm{d})$, pitavastatin $(0.5 \mathrm{mg} / \mathrm{kg} / \mathrm{d})$, valsartan $(5 \mathrm{mg} / \mathrm{kg} / \mathrm{d})$, and pitavastatin and valsartan in combination (top); and densitometric analysis (bottom; $n=6$ ). Data are expressed as the mean $\pm S E M . \quad * p<0.01$ vs. the vehicle (control) and eplerenone groups.

\section{Change in Vascular Nitrotyrosine}

The vascular nitrotyrosine measured as a surrogate index of vascular peroxynitrite level was significantly lower in the drug-treated groups, with the exception of the hydralazine group, than in the control group (Fig. 4). The pitavastatin plus valsartan combination produced a significantly lower level of vascular nitrotyrosine $(0.41 \pm 0.02 \mathrm{pmol} / \mathrm{mg}$ protein $)$ than either pitavastatin $(0.73 \pm 0.05 \mathrm{pmol} / \mathrm{mg}$ protein $)$, or valsartan $(0.80 \pm 0.04 \mathrm{pmol} / \mathrm{mg}$ protein) alone $(p<0.01$ each $)$. These results demonstrate the additive effect of pitavastatin plus valsartan for reducing peroxynitrite and for reducing the reaction 
A
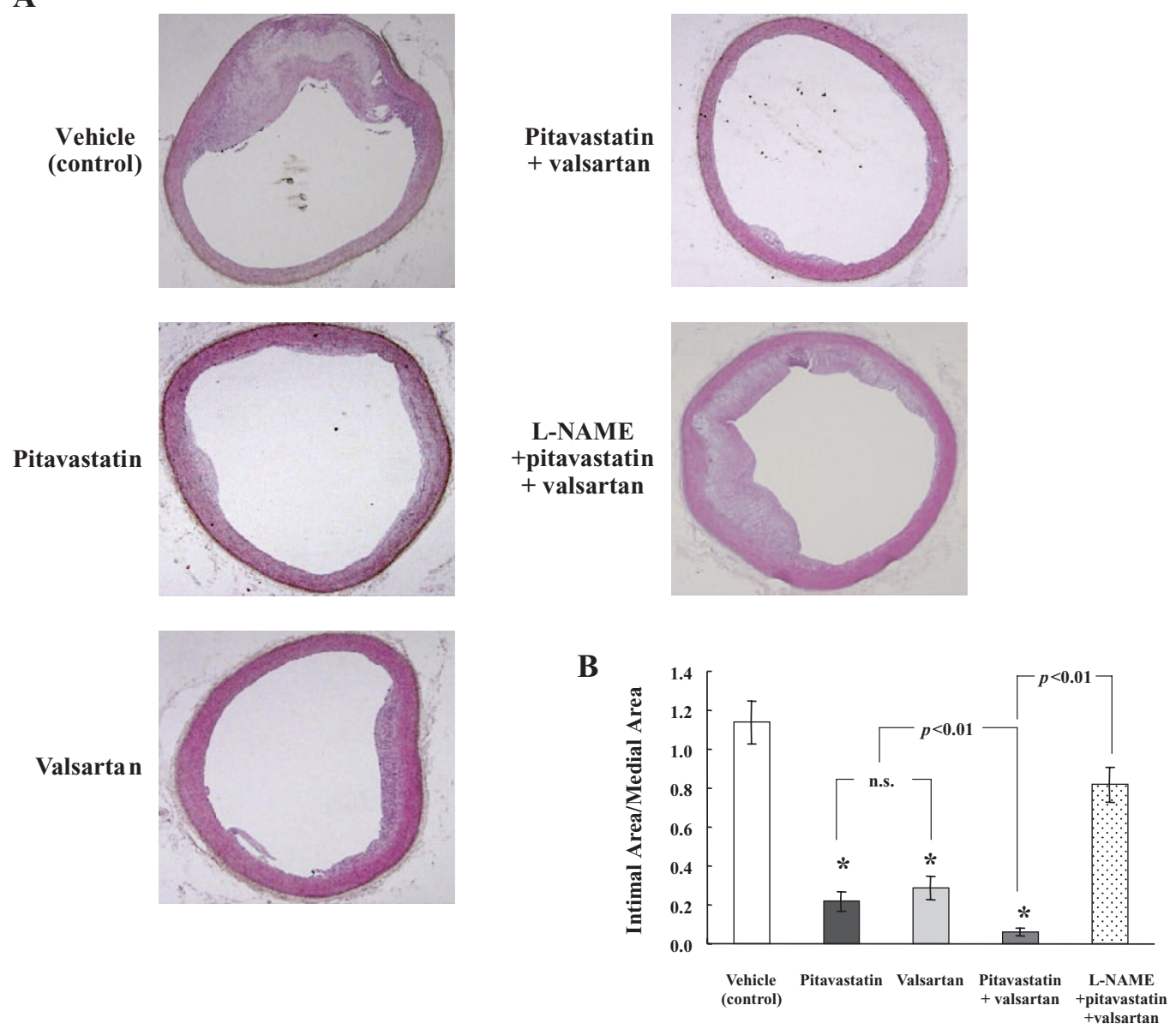

Fig. 6. A: Photomicrographs of cross-sections of the thoracic aorta. The sections were stained with hematoxylin and eosin for thoracic aorta of WHHLMI rabbits treated with vehicle (control), pitavastatin $(0.5 \mathrm{mg} / \mathrm{kg} / \mathrm{d})$, valsartan $(5 \mathrm{mg} / \mathrm{kg} / \mathrm{d})$, and pitavastatin and valsartan in combination without or with L-NAME. B: Mean intimal area/medial area ratio of the thoracic aorta compared among groups of WHHLMI rabbits treated with vehicle (control), pitavastatin $(0.5 \mathrm{mg} / \mathrm{kg} / \mathrm{d})$, valsartan $(5 \mathrm{mg} / \mathrm{kg} / \mathrm{d})$, and pitavastatin and valsartan in combination without or with L-NAME. Data are expressed as the mean $\pm S E M(n=6) .{ }^{*} p<0.01 \mathrm{vs}$. control.

between superoxide and NO resulting in increased NO bioavailability.

\section{Vascular eNOS Protein Expression}

Vascular eNOS protein expression was significantly higher in the drug-treated groups, with the exception of the hydralazine group, than in the control group (Fig. 5). The pitavastatin-valsartan combination produced a significantly higher level of vascular eNOS protein expression (Fig. 5).

\section{Atherosclerotic Plaque Formation}

Atherosclerotic plaque formation was observed in a typical histological section of the thoracic aorta from a WHHLMI rabbit. Compared to the control, pitavastatin or valsartan alone markedly attenuated the formation of atherosclerotic plaque. Combined administration of pitavastatin and valsartan further inhibited the plaque formation compared with pitavastatin or valsartan alone as shown in Fig. 6A. The atherosclerotic change was quantified by calculating the ratio of the intimal area/medial area in the section as shown in Fig. $6 \mathrm{~B}$. The ratio was smaller with pitavastatin or valsartan alone than with the control, and was decreased further by combined administration of pitavastatin and valsartan. Interestingly, cotreatment with L-NAME significantly abolished the plaque suppression induced by pitavastatin plus valsartan (Fig. 6B). Furthermore, OCT technology demonstrated that lipid-rich plaque (lipid content $>2$ quadrants) in any of the images was only found in the control group (Fig. 7A). The measured degree of lipid arc along the thoracic aorta was significantly reduced in the pitavastatin and valsartan combination group 
A

a

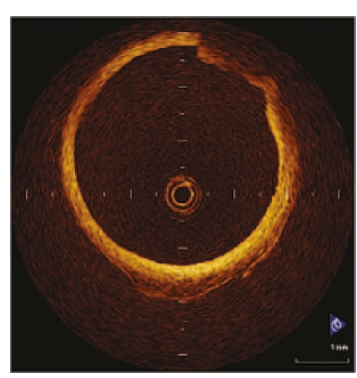

b

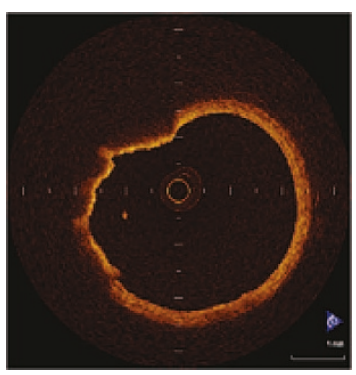

c

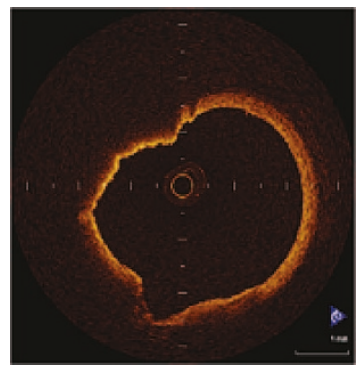

d

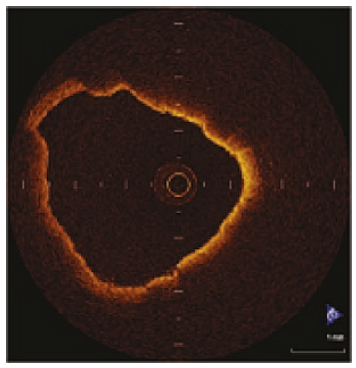

B

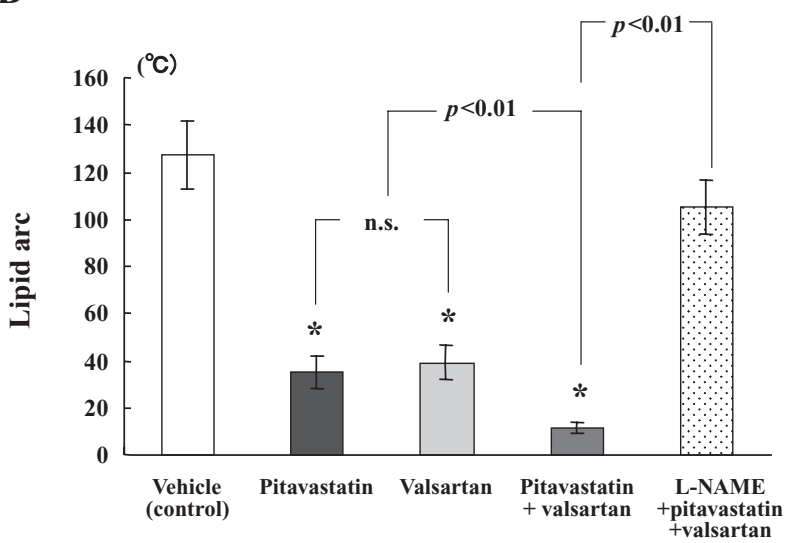

Fig. 7. OCT image of the thoracic aorta in WHHLMI rabbits. A: Lipid content was classified into 4 grades according to the number of involved quadrants on cross-sectional OCT images; that is, 1 quadrant (a), 2 quadrants (b), 3 quadrants (c), and 4 quadrants (d). Lipid-rich plaque (lipid content $>2$ quadrants) in any of the images was found only in the vehicle (control) group. B: Degree of lipid arc on the cross-sectional OCT image in involved segments along the thoracic aorta compared among groups of WHHLMI rabbits treated with vehicle (control), hydralazine $(10 \mathrm{mg} / \mathrm{kg} / \mathrm{d})$, pitavastatin $(0.5$ $\mathrm{mg} / \mathrm{kg} / \mathrm{d})$, valsartan $(5 \mathrm{mg} / \mathrm{kg} / \mathrm{d})$, and pitavastatin and valsartan in combination without or with L-NAME. Plaque lesions were quantified as the degree of lipid arc in involved segments along the thoracic aorta using cross-sectional OCT imaging. Data are expressed as the mean \pm SEM $(n=6$, each). ${ }^{*} p<0.01$ vs. control. compared with the groups treated with pitavastatin or valsartan alone, which effect was significantly abolished by cotreatment with L-NAME (Fig. 7B).

\section{Discussion}

The present study provides the first evidence that the administration of either pitavastatin or valsartan increased both ACh-induced and basal plasma NO concentrations in WHHLMI rabbits. More intriguingly, combined treatment with both pitavastatin and valsartan significantly increased ACh-induced as well as basal plasma NO concentration beyond the levels achieved by either agent alone.

Several clinical studies have demonstrated that both HMGCoA inhibitors and ARBs improve endothelial dysfunction in patients with cardiovascular risk factors or coronary artery disease (14-17). However, in those studies the endothelial function was been monitored by change in NO bioavailability as measured almost exclusively by secondary effects of NO, such as biologically inactive products of NO (nitrite and nitrate) or flow-dependent endothelium-mediated vasodilation. In this study, we provided direct evidence that co-treatment with a statin and an ARB significantly increased AChinduced as well as basal plasma NO concentration compared with either statin or ARB alone.

In our previous study, the direct in vivo endotheliumderived NO was measured with a catheter-type NO sensor in a rabbit model (8). The NO sensor was shown to be stable and specific to NO, because direct exposure to either ACh or LNMMA solution did not cause any significant change in the baseline current (data not shown). The NO sensor also showed no noticeable change in the baseline current due to solution mixing, suggesting that it was not affected by fluid (blood) motion (data not shown). Thus, this system has high specificity for NO, and the monitored current reflected the change in plasma concentration of NO released from the endothelium after ACh and L-NMMA infusion.

There are several potential mechanisms that could account for the impairment in NO bioavailability with statins and ARBs. First, this study showed that both pitavastatin and valsartan increase eNOS protein levels in the aorta of WHHLMI rabbits. Interestingly, the combination of pitavastatin and valsartan produced a significantly higher level of vascular eNOS protein, with the two agents probably using different molecular mechanisms. This could be one of the reasons why the combination of pitavastatin and valsartan exerts additional effects on NO production. Secondly, Tao et al. have demonstrated that hypercholesterolemia induces endothelial dysfunction by increased $\mathrm{O}_{2}^{-}$generation rather than decreased NO production (18). On the other hand, Ang II and AT1 receptor activation stimulate NADPH oxidase, resulting in the generation of reactive oxygen species (ROSs) in vascular cells and eventually, endothelial dysfunction (19-21). Interestingly, Warnholtz et al. (22) have demonstrated using WHHLMI rabbits that hypercholesterolemia is associated 
with AT1-receptor upregulation and increased $\mathrm{O}_{2}^{-}$production secondary to an activation of vascular NADPH oxidase. This study showed that both pitavastatin and valsartan inhibited NADH/NADPH oxidase activity in WHHLMI rabbits. Although it is tempting to speculate that statins would inhibit NADPH oxidase activity at least in part through a mechanism secondary to reduced hypercholesterolemia, there is no direct evidence confirming this hypothesis. Further studies will be needed on this important subjects. Emerging evidence suggests that in addition to oxidative stress, nitrosative stress also plays a significant role in tissue injury, including injury of the endothelium (23). In the present study, our results showed that either pitavastatin or valsartan treatment significantly inhibits the production of vascular peroxynitrite $\left(\mathrm{ONOO}^{-}\right)$, a ROSs generated from a rapid reaction of $\mathrm{NO}$ with superoxide in WHHLMI rabbits. More importantly, the combined treatment with pitavastatin and valsartan significantly reduced plasma peroxynitrite concentrations compared with monotherapy. Both superoxide $\left(\mathrm{O}_{2}^{-}\right)$and $\mathrm{ONOO}^{-}$have been demonstrated to oxidize $\mathrm{BH}_{4}$, a critical eNOS cofactor, and thereby lead to eNOS uncoupling (24). Interestingly, Laursen et al. (25) have shown that peroxynitrite is much more potent than $\mathrm{O}_{2}{ }^{-}$in oxidizing tetahydrobiopterin. During eNOS uncoupling, electron flow through the enzyme results in reduction of molecular oxygen at the prosthetic heme site rather than formation of NO (26). If this phenomenon occurred in vivo, it could result in increased production of ROSs, including peroxynitrite, and reduced NO bioavailability. Taken together, our results suggest that increased production of peroxynitrite would at least in part result in diminished NO bioavailability through the breakdown of $\mathrm{NO}$ in WHHLMI rabbits.

In the present study, histological and OCT studies demonstrated that both a statin and an ARB reduced plaque area. More importantly, the combined treatment with the statin and ARB dramatically reduced the plaque area accompanied with marked suppression of the production of vascular peroyxnitrite. Dysfunction of eNOS has also been shown to accelerate atherosclerotic lesion formation in mice (27), whereas overexpression of eNOS in mice with hypercholesterolemia resulted in increased eNOS-derived $\mathrm{O}_{2}^{-}$production and promotion of atherogenesis (28). In fact, this study demonstrated that co-treatment with L-NAME significantly abolished the plaque suppression induced by the combination of pitavastatin and valsartan. These results are compatible with previous reports. Horiuchi et al. (29) have demonstrated that co-treatment with valsartan and fluvastatin significantly inhibits neointimal formation induced by cuff placement around the femoral artery. They also postulated that the cholesterol-independent inhibition of AT1 receptor-mediated signaling by statins demonstrates a novel regulating mechanism that may contribute to the beneficial effects of these drugs beyond the mere lowering of plasma cholesterol, especially when combined with an ARB. In addition, Li et al. (30) have shown that treatment with even a low dose of valsartan, together with a low dose of fluvastatin that did not influence blood pressure or plasma cholesterol, effectively attenuated the size of atherosclerotic lesions and the lipid deposition with a decrease in oxidative stress and vascular inflammation in apolipoprotein E null mice treated with a high-cholesterol diet for 10 weeks.

The unique capability of OCT to quantify the size of the lipid-rich plaque area and the thickness of the cap has been clearly confirmed by several groups $(11,31,32)$. Because OCT systems allow screening of long arterial segments, the effects of drugs on atherosclerotic change along the thoracic aorta could be examined thoroughly by this method.

In conclusions, this study has shown that combined treatment with a statin and an ARB may have beneficial effects on NO bioavailability using a catheter-type NO sensor as well as on vascular remodeling evaluated by histology and OCT in WHHLMI rabbits.

\section{References}

1. Libby P, Theroux P: Pathophysiology of coronary artery disease. Circulation 2005; 111: 3481-3488.

2. Kannel WB: Risk stratification in hypertension: new insights from the Framingham Study. Am J Hypertens 2000; 13: $3 \mathrm{~S}-10 \mathrm{~S}$.

3. Ginsberg HN, Stalenhoef AF: The metabolic syndrome: targeting dyslipidemia to reduce coronary risk. J Cardiovasc Risk 2003; 10: 121-128.

4. Sowers JR: Effects of statins on the vasculature. Implications for aggressive lipid management in the cardiovascular metabolic syndrome. Am J Cardiol 2003; 91: 14B-22B.

5. Kjeldsen SE, Julius S: Hypertension mega-trials with cardiovascular and points: effect of angiotensin-converting enzyme inhibitors and angiotensin receptor blockers. Am Heart J 2004; 148: 747-754.

6. Mochizuki S, Miyasaka T, Goto M, et al: Measurement of acetylcholine-induced endothelium-derived nitric oxide in aorta using a newly developed catheter-type nitric oxide sensor. Biochem Biophys Res Commun 2003; 306: 505-508.

7. Neishi Y, Mochizuki S, Miyasaka T, et al: Evaluation of bioavailability of nitric oxide in coronary circulation by direct measurement of plasma nitric oxide concentration. Proc Natl Acad Sci U S A 2005; 102: 11456-11461.

8. Imanishi T, Kobayashi K, Kuroi A, et al: Effects of angiotensin II on NO bioavailability evaluated using a cathetertype NO sensor. Hypertension 2006; 48: 1058-1065.

9. Nickenig G: Should angiotensin II receptor blockers and statins be continued? Circulation 2004; 110: 1013-1020.

10. Suzuki H, Yamazaki H, Aoki T, et al: Lipid-lowering and antiatherosclerotic effect of NK-104, a potent 3-hydroxy-3methylglutaryl coenzyme A reductase inhibitor, in Watanabe heritable hyperlipidemic rabbits. Arzneimittelforschung 2000; 50: 995-1003.

11. Ter Steege JCA, Koster-Kamphuis L, Van Straaten EA, et al: Nitrotyrosine in plasma of celiac disease patients as detected by a new sandwich ELISA. Free Radic Biol Med 1998; 25: 953-963.

12. Jang IK, Tearney GJ, MacNeill B, et al: In vivo characterization of coronary atherosclerotic plaque by use of optical 
coherence tomography. Circulation 2005; 111: 1551-1555.

13. Imanishi T, McBride J, Ho Q, O’Brien KD, Schwartz SM, Han DK: Expression of cellular FLICE-inhibitory protein in human coronary arteries and in a rat vascular injury model. Am J Pathol 2000; 156: 125-137.

14. Balk EM, Karas RH, Jordan HS, Kupelnick B, Chew P, Lau $\mathrm{J}$ : Effects of statins on vascular structure and function; a systemic review. Am J Med 2004; 117: 775-790.

15. O'Driscoll G, Green D, Rankin J, Stanton K, Taylor R: Improvement in endothelial function by angiotensin converting enzyme inhibition in insulin-dependent diabetes mellitus. J Clin Invest 1997; 100: 678-684.

16. Cheetham C, Collis J, O’Driscoll G, Stanton K, Taylor R, Green D: Losartan, an angiotensin type 1 receptor antagonist, improves endothelial function in non-insulin-dependent diabetes. J Am Coll Cardiol 2000; 36: 1461-1466.

17. Hornig B, Lnadmesser U, Kohler C, et al: Comparative effect of ACE inhibition and angiotensin II type 1 receptor antagonist on bioavailability of nitric oxide in patients with coronary artery disease: role of superoxide dismutase. Circulation 2001; 103: 799-805.

18. Tao L, Liu HR, Gao E, et al: Antioxidative, antinitrative, and vasculoprotective effects of a peroxisome proliferatoractivated receptor- $\gamma$ agonist in hypercholesterolemia. Circulation 2003; 108: 2805-2811.

19. Griendling KK, Minieri CA, Ollerenshaw JD, Alexander RW: Angiotensin II stimulates NADH and NADPH oxidase activity in cultured vascular smooth muscle cells. Circ Res 1994; 74: 1141-1148.

20. Rajagopalan S, Kurz S, Munzel T, et al: Angiotensin IImediated hypertension in the rat increases vascular superoxide production via membrane NADH/NADPH oxidase activation. J Clin Invest 1996; 97: 1916-1923.

21. Pagano PJ, Clark JK, Cifuentes-Pagano ME, Clark SM, Callis GM, Quinn MT: Localization of a constitutively active, phagocyte-like NADPH oxidase in rabbit aortic adventitia: enhancement by angiotensin II. Proc Natl Acad Sci U S A 1997; 94: 14483-14488.

22. Warnholtz A, Nickenig G, Schulz E, et al: Increased
NADH-oxidase-mediated superoxide production in the early stages of atherosclerosis: evidence for involvement of the renin-angiotensin system. Circulation 1999; 99: 20272033.

23. Griendling KK, FitzGerald GA: Oxidative stress and cardiovascular injury: Part I: basic mechanism and in vivo monitoring of ROS. Circulation 2003; 108: 1912-1916.

24. Landmesser U, Dikalov S, Price SR, et al: Oxidation of tetrahydropterin leads to uncoupling of endothelial cell nitric oxide synthesis in hypertension. J Clin Invest 2003; 111: 1201-1209.

25. Laursen JB, Somers M, Kurz S, et al: Endothelial regulation of vasomotion in apoE-deficient mice: implications for interactions between peroxynitrite and tetrahydrobiopterin. Circulation 2001; 103: 1282-1288.

26. Stuehr D, Pou S, Rosen GM: Oxygen reduction by nitric oxide synthases. J Biol Chem 2001; 276: 145333-145336.

27. Kawashima S, Yokoyama M: Dysfunction of endothelial nitric oxide synthase and atherosclerosis. Arterioscler Thromb Vasc Biol 2004; 24: 998-1005.

28. Ozaki M, Kawashima S, Yamashita T, et al: Overexpression of endothelial nitric oxide synthase accelerates atherosclerotic lesion formation in apo E-deficient mice. J Clin Invest 2002; 110: 331-340.

29. Horiuchi M, Cui TX, Li Z, Li JM, Nakagami H, Iwai M: Fluvastatin enhances the inhibitory effects of a selective angiotensin II type 1 receptor blocker, valsartan, on vascular neointimal formation. Circulation 2003; 107: 106-112.

30. Li Z, Iwai M, Wu L, et al: Fluvastatin enhances the inhibitory effects of a selective AT1 receptor blocker, valsartan, on atherosclerosis. Hypertension 2004; 44: 758-763.

31. Jang IK, Bouma BE, Kang DH, et al: Visualization of coronary atherosclerotic plaques in patients using optical coherence tomography: comparison with intravascular ultrasound. J Am Coll Cardiol 2002; 39: 604-609.

32. Yabushita H, Bouma BE, Houser SL, et al: Characterization of human atherosclerosis by optical coherence tomography. Circulation 2002; 106: 1640-1645. 